\title{
Technè
}

La science au service de l'histoire de l'art et de la préservation des biens culturels

$45 \mid 2017$

Bronzes grecs et romains : études récentes sur la statuaire antique

\section{Étude interdisciplinaire des naïskoi du Musée d'Histoire de Marseille}

Apports à la connaissance de la sculpture massaliote de l'époque archaïque

Interdisciplinary study of the naïskoi in the Museum of the History of Marseille: towards a better knowledge of the sculpture production in Marseille during the Archaic period

Nicolas Bouillon, Philippe Bromblet, Odile Guillon et Laura Rohaut

\section{(2) OpenEdition}

Journals

Édition électronique

URL : http://journals.openedition.org/techne/1380

DOI : 10.4000/techne. 1380

ISSN : 2534-5168

Éditeur

C2RMF

\section{Édition imprimée}

Date de publication : 1 mai 2017

Pagination : 136-142

ISBN : 978-2-7118-6408-9

ISSN : 1254-7867

Référence électronique

Nicolas Bouillon, Philippe Bromblet, Odile Guillon et Laura Rohaut, « Étude interdisciplinaire des naïskoi du Musée d'Histoire de Marseille », Technè [En ligne], 45 | 2017, mis en ligne le 19 décembre 2019, consulté le 22 juillet 2020. URL : http://journals.openedition.org/techne/1380 ; DOI : https://doi.org/ 10.4000/techne.1380

La revue Technè. La science au service de l'histoire de l'art et de la préservation des biens culturels est mise à disposition selon les termes de la Licence Creative Commons Attribution - Pas d'Utilisation Commerciale - Pas de Modification 4.0 International. 


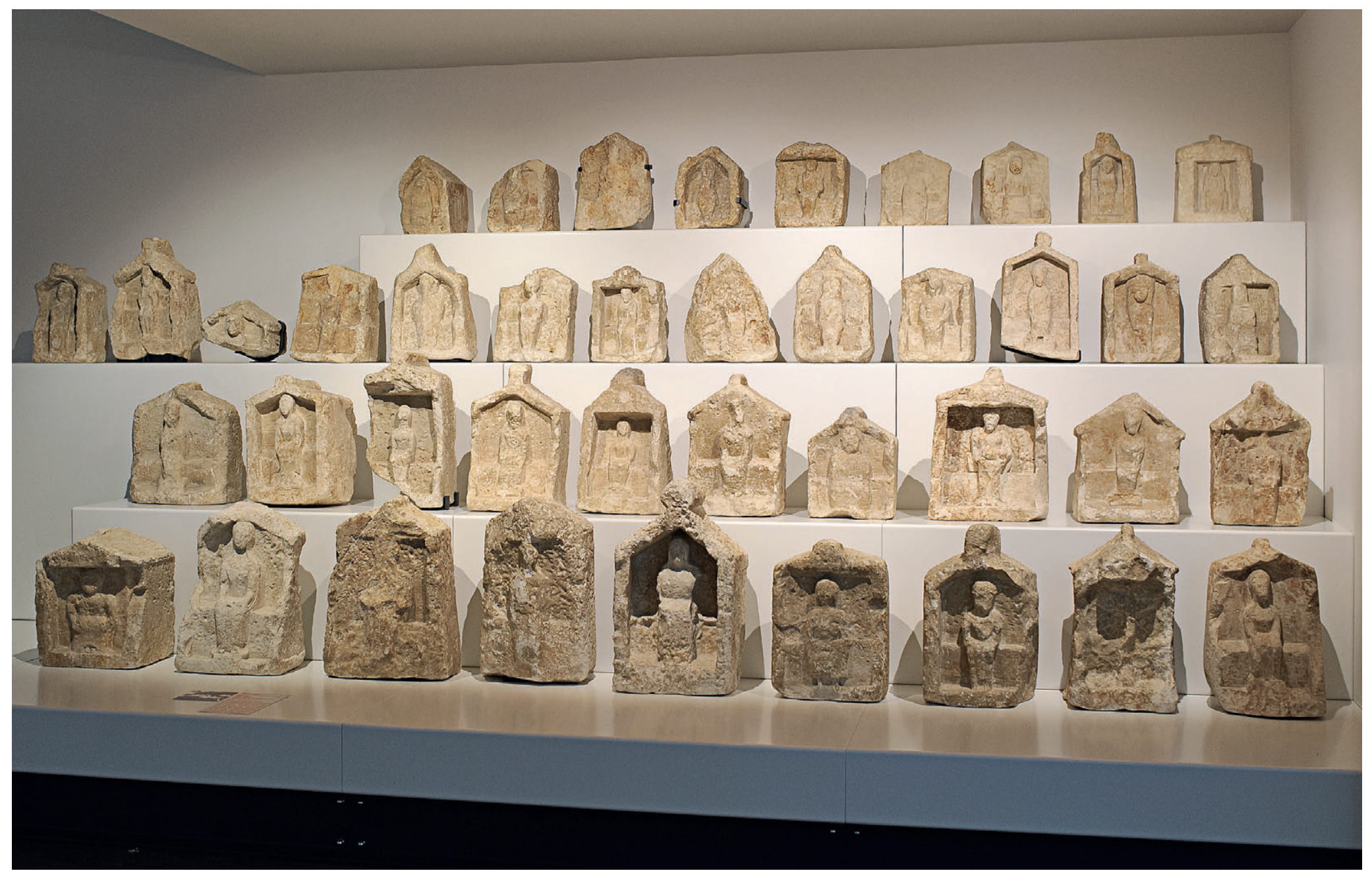

Fig. 1. Vue générale des naïskoi exposés au Musée d'Histoire de Marseille. @ O. Guillon. 
Nicolas Bouillon

Philippe Bromblet

Odile Guillon

Laura Rohaut

\section{Étude interdisciplinaire des nä̈skoi du Musée d'Histoire de Marseille : apports à la connaissance de la sculpture massaliote de l'époque archaïque}

Interdisciplinary study of the naïskoi in the Museum of the History of Marseille: towards a better knowledge of the sculpture production in Marseille during the Archaic period
Résumé. Le présent article expose les résultats des analyses pétrographiques et physico-chimiques menées sur les petits édicules archaïques en calcaire, pour certains peints, découverts au $X I X^{e}$ siècle à Marseille, grâce à une collaboration interdisciplinaire mise en place dans le cadre d'une étude archéologique.

Mots-clés. Naïskos, sculpture, période archaïque, Marseille, calcaire, polychromie.
Abstract. The present paper presents the results of petrographic and physico-chemical analyses carried out on small Archaic limestone aedicula - some of which were painted - discovered in Marseille in the 19th century. This interdisciplinary, collaborative project was set up within the framework of an archaeological study.

Keywords. Naiskos, sculpture, Archaic period, Marseille, limestone, polychromy.
La cité grecque de Marseille a offert un ensemble exceptionnel de quarante-quatre petits édicules en calcaire, datés au plus tôt du troisième quart du VI ${ }^{\mathrm{e}}$ siècle avant J.-C., qui représentent chacun une divinité féminine, de style ionien archaïque, assise sur une banquette dans la niche d'un naïskos ("petit temple " ou « chapelle » en grec) (fig. 1). Mis au jour sous le sol de l'ancienne rue Négrel en 1863 et en 1946, ce matériel conservé au Musée d'Histoire de la ville a fait l'objet de nombreux commentaires et débats ayant principalement porté sur l'identité de la déesse ${ }^{1}$, mais ni la pierre, ni les restes de couleur n'avaient à ce jour fait l'objet d'analyses. Ainsi, s'agissait-il de proposer une étude scientifique approfondie, fondée sur l'apport inédit d'analyses des matériaux constitutifs, afin de porter un regard nouveau sur une collection anciennement trouvée. Les résultats de cette étude, menée entre 2012 et 2017 grâce à une collaboration interdisciplinaire, sont présentés ici².

\section{Analyse pétrographique}

Une étude pétrographique a été réalisée pour confirmer la nature et la provenance de la pierre constitutive des naïskoi. Les identifications précédentes, effectuées à l'œil nu, avaient déterminé un calcaire local de consistance relativement tendre, apparenté à la craie d'Allauch ${ }^{3}$, et plus récemment au calcaire de Saint-Victor ${ }^{4}$, dont des carrières antiques, datées de l'époque hellénistique (aujourd'hui inaccessibles), ont été localisées sous l'ancienne abbaye ${ }^{5}$.

\section{Méthodologie}

Cinq naïskoi ont été échantillonnés sur des plans de cassure peu visibles. Les fragments bruts ont d'abord été décrits sous la loupe binoculaire, puis des lames minces ont été confectionnées pour une analyse pétrographique au microscope optique polarisant. Les prélèvements ont été comparés, d'une part, à trois échantillons de référence de la lithothèque du CICRP, prélevés dans les cryptes médiévales de l'ancienne abbaye de Saint-Victor sur un front de taille du XII ${ }^{\mathrm{e}}$ siècle et sur deux piliers du XII ${ }^{\mathrm{e}}$ ou XIII ${ }^{\mathrm{e}}$ siècle; et d'autre part, à trois objets, datés des $\mathrm{VI}^{\mathrm{e}}$ et $\mathrm{V}^{\mathrm{e}}$ siècles avant J.-C., conservés au Musée d'Histoire, et réputés être en calcaire de Saint-Victor (un chapiteau ionique, un bloc avec graffiti et un bloc inscrit).

\section{Résultats}

Les naïskoi ont été taillés dans un calcaire blanchâtre tendre à pâte fine (mudstone) parsemée parfois de quelques amas ferrugineux orangés. En lame mince, la roche montre une matrice calcitique finement cristallisée (micrite) rarement homogène. Elle forme soit une structure péloïdale avec des grains arrondis ou allongés, parfois anguleux, assemblés de manière plus ou moins compacte (fig. 2), soit une structure grumeleuse qui présente des amas micritiques ménageant des vides intergranulaires plus ou moins importants, remplis par une microsparite (fig. 3). Cette variation de texture existe sur le même échantillon sous la forme de passées

Nicolas Bouillon, ingénieur chimiste, Centre Interdisciplinaire de Conservation et Restauration du Patrimoine (nicolas.bouillon@cicrp.fr). Philippe Bromblet, géologue, CICRP (philippe.bromblet@cicrp.fr). Odile Guillon, photographe-radiologue, CICRP (odile.guillon@cicrp.fr). Laura Rohaut, docteur en archéologie grecque, Aix-Marseille Université, UMR 7299 Centre Camille-Jullian (laura.rohaut@gmail.com). 


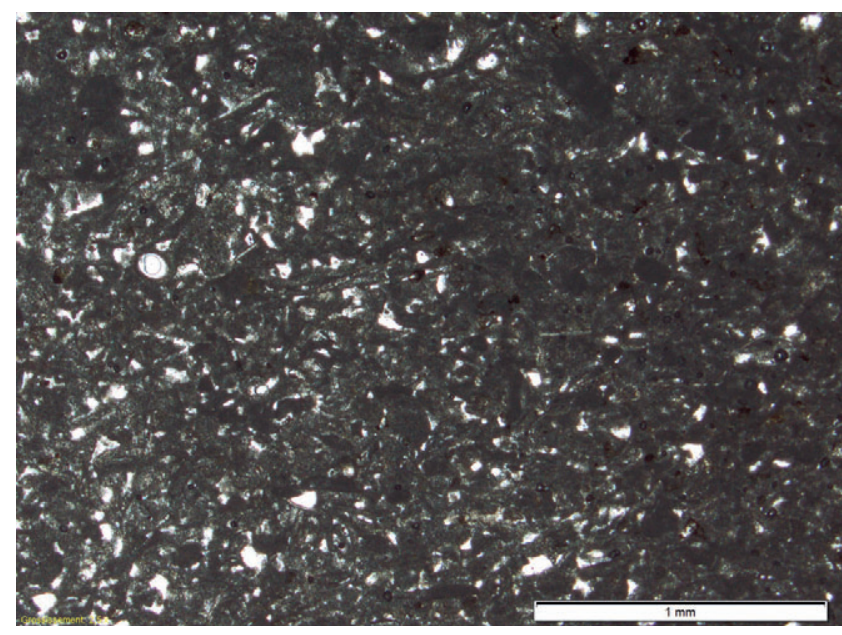

Fig. 2. Microfaciès du naïskos inv. MHM 83.7.593 en lame mince (lumière polarisée, non analysée x 25). Micrite à structure grumeleuse. (C) Ph. Bromblet.

millimétriques. La porosité vacuolaire (fenestrae) est faible ou nulle. Des fragments de microfossiles de gastéropodes et de crustacés à fine valve (ostracodes), caractéristiques d'un dépôt de milieu lacustre, sont inclus en quelques exemplaires dans cette matrice. Les grains de quartz sont rares et fin $(<0,2 \mathrm{~mm})$.

Les échantillons de référence sont constitués d'un calcaire comparable, mais on note la présence de nombreux fragments algaires rosés dans la pierre du chapiteau ionique et de quelques characées (tiges en coupe de végétaux d'eau douce ou saumâtre) dans deux échantillons issus des cryptes médiévales (pilier et front de taille). Les nombreux traits communs à tous les échantillons indiquent qu'ils appartiennent à un même faciès calcaire lacustre ou palustre qui peut être assimilé au calcaire de Saint-Victor. Les différences minimes observées ne traduisent probablement que des petites variations latérales ou verticales des conditions de dépôt (profondeur, agitation, salinité...). Les études pétrographiques valident donc l'hypothèse déjà formulée sur l'origine locale de la pierre. Le calcaire de Saint-Victor appartient à la formation géologique des calcaires de l'Estaque d'âge Stampien (Oligocène inférieur) ${ }^{6}$. Deux faciès sont reportés : le plus courant est massif, le second, plus rare, est laminé. La hauteur des bancs est limitée à quelques dizaines de centimètres. Les sculptures sont toutes taillées dans des blocs homogènes, en délit, ce qui correspondrait effectivement à des hauteurs de banc limitées (20 à $30 \mathrm{~cm}$ au maximum).

\section{Caractérisation des restes de polychromie}

Onze naïskoi présentent des traces de peinture. L'examen visuel minutieux de chacune des pièces révèle que les couleurs subsistantes sont concentrées exclusivement sur la face antérieure des édicules. L'interprétation des photographies haute résolution effectuées sur les pièces sélectionnées pour l'étude confirme ces observations. La couleur majoritairement conservée est le rouge dont les traces apparaissent sur le cadre, dans

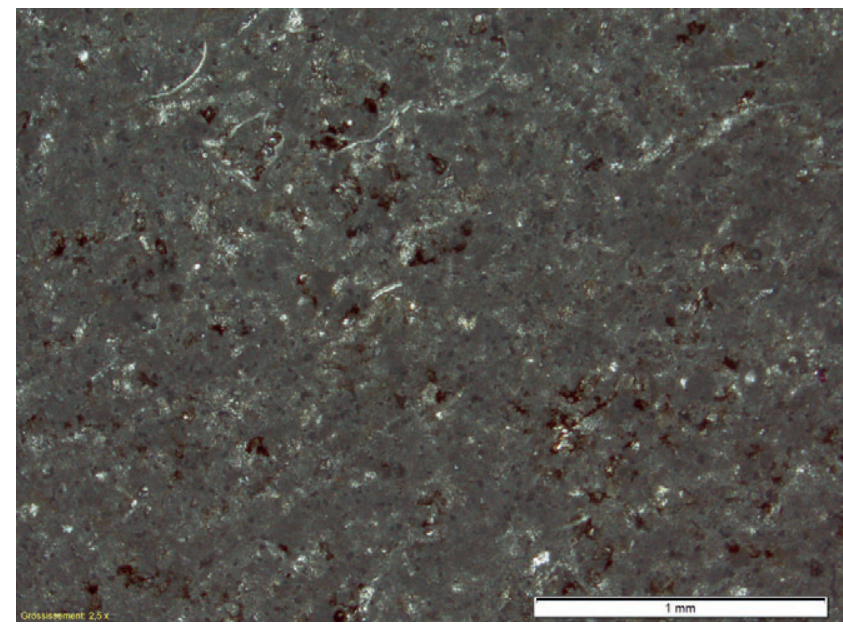

Fig. 3. Microfaciès du naïskos inv. MHM 83.7.592 en lame mince (lumière polarisée, non analysée x 25). Micrite à structure péloïdale. (C) $\mathrm{Ph}$. Bromblet.

le fond de la niche, sur le vêtement de la divinité (chitôn et voile), sur l'assise et le devant de la banquette où figure dans certains cas un décor de frange dans la partie supérieure (fig. 4). Deux types de peinture rouge ont été observés : l'un possède une teinte très ténue et un état de surface fin, l'autre possède une teinte plus franche et un aspect de surface plus granuleux (fig. 5 et 6 ). Des traces de couleur noire ont été observées sur le devant de la banquette d'un des naïskoi sur lequel subsiste également des restes très ténus de franges rouges. On peut également suspecter la présence de jaune dans cette même zone (fig. 7). Le bleu - semble-t-il observé au début du $\mathrm{XX}^{\mathrm{e}}$ siècle ${ }^{7}-$ n'a laissé aucune trace. On notera que lorsqu'ils étaient conservés au musée du château Borély, les naïskoi ont subi des arrosages fréquents d'eau javellisée lors des nettoyages des locaux ${ }^{8}$. Une campagne de consolidation de la pierre a été programmée dans les années 1960 et une partie des édicules a été traitée par un produit biocide dont nous ne connaissons que le nom MOS-monument ${ }^{9}$. Nous ignorons aujourd'hui quels ont été les effets sur les sculptures.

Les restes de peintures ne doivent pas être confondus avec les altérations colorées qui apparaissent sous la forme de croûtes, plus ou moins épaisses, ou de taches de couleur rouille. Leur localisation aléatoire en surface de zones cassées ou érodées et leur composition chimique permettent de les différencier ${ }^{10}$.

\section{Méthodologie}

Une étude documentaire, incluant la consultation des publications antérieures et des archives photographiques, a constitué un préalable nécessaire à l'étude matérielle. Dans un second temps, l'élaboration d'un relevé précis de l'ensemble des traces colorées a été complétée par un dossier d'imagerie scientifique (photographies en lumière directe et semi-rasante, dans l'infrarouge, sous ultraviolet) mettant en évidence certaines réactions spécifiques des matériaux en surface. Les acquisitions d'image ont été réalisées après un nettoyage superficiel des stèles qui 

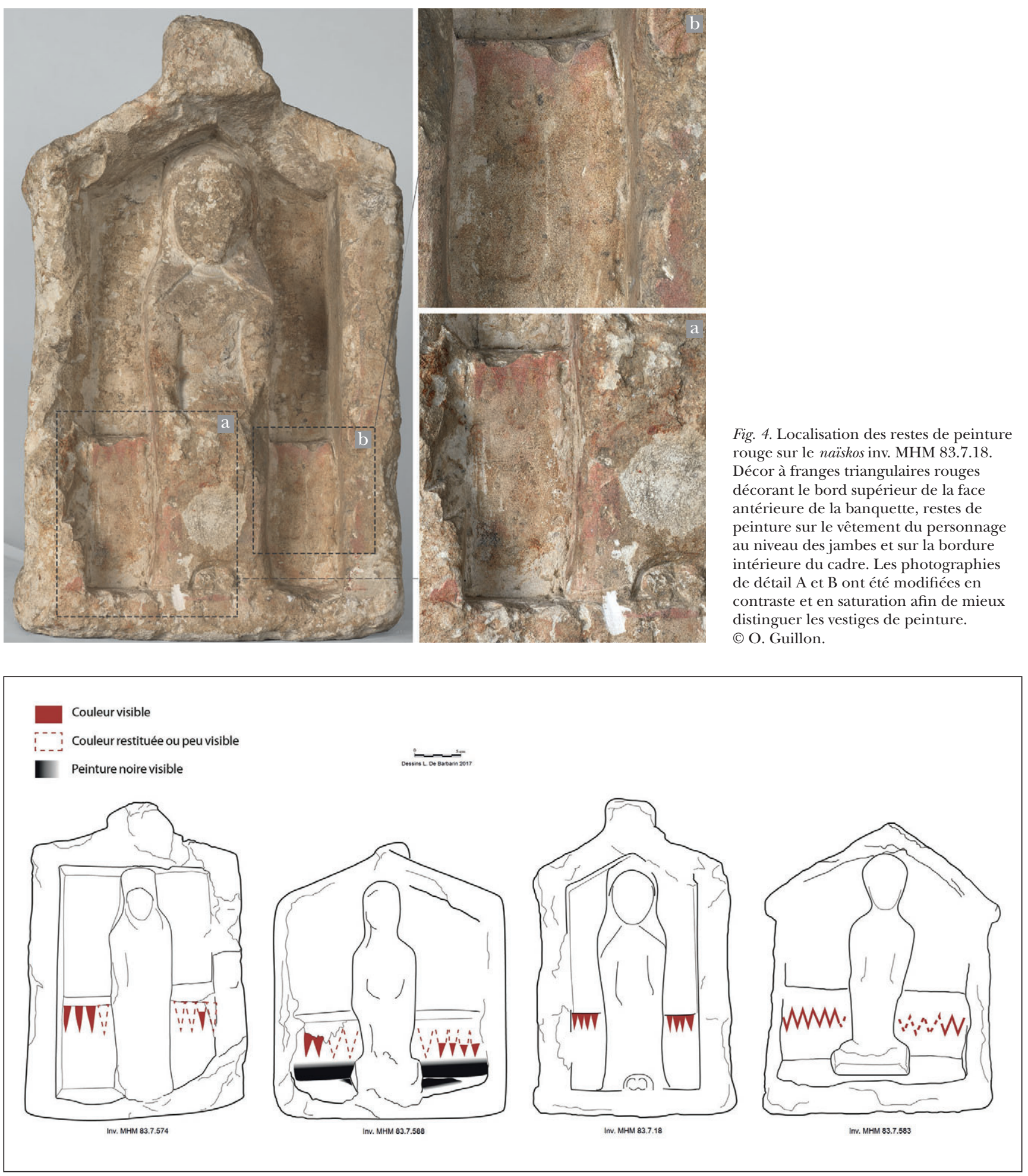

Fig. 5. Dessins de restitution des quatre décors de franges. (C L. De Barbarin, 2017.

a été mené dans le cadre du chantier des collections par Julia Greiner, restauratrice de sculptures, sous la conduite de Solange Rizoulières, conservateur. Ce nettoyage a principalement consisté à soigneusement dépoussiérer l'ensemble des stèles à l'aide d'un pinceau souple et d'un aspirateur. Quelques surfaces plus encrassées ont été nettoyées par microsablage (pression max 0,2 bar, poudre de silicate d'alumine, diamètre moyen
$20 \mu \mathrm{m})$. L'identification des pigments et la caractérisation des techniques d'application ont été réalisées par des analyses non-destructives en micro-fluorescence X (MFX) et à partir des coupes stratigraphiques d'un nombre minimum de microprélèvements de peinture, effectués au scalpel en bordure de lacunes dans des zones peu visibles. Les cartographies X élémentaires au MEB/EDS ont permis d'identifier et de localiser
Fig. 4. Localisation des restes de peinture rouge sur le naïskos inv. MHM 83.7.18. Décor à franges triangulaires rouges décorant le bord supérieur de la face antérieure de la banquette, restes de au niveau des jambes et sur la bordure intérieure du cadre. Les photographies de détail A et B ont été modifiées en contraste et en saturation afin de mieux distinguer les vestiges de peinture. (C) O. Guillon. 

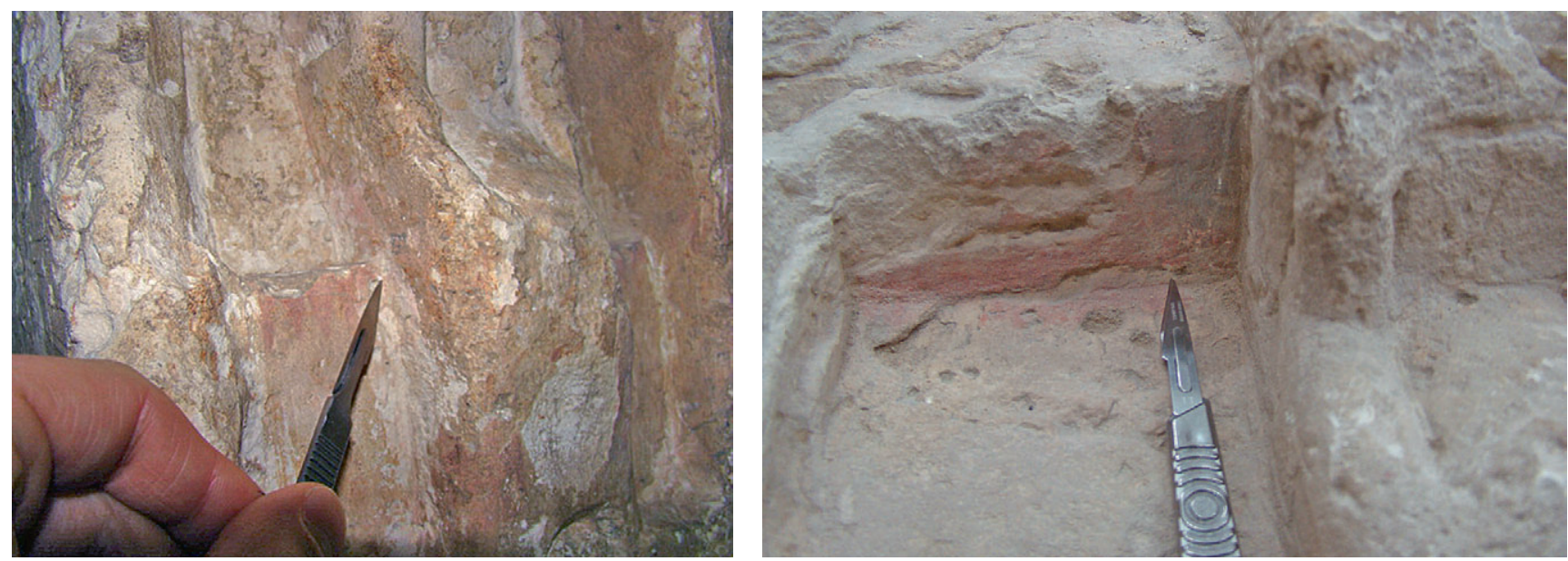

Fig. 6. Macrophotographies des restes de peinture rouge sur le naïskos inv. MHM 83.7.18 (à gauche - profil stratigraphique $\mathrm{n}^{\circ}$ 1) et sur le naïskos inv. MHM 83.7.581 (à droite - profil stratigraphique $\mathrm{n}^{\circ}$ 2). Les restes de peinture rouge à droite présentent à l'œil nu une teinte légèrement plus vive et un aspect plus granuleux. $\odot \mathrm{N}$. Bouillon.

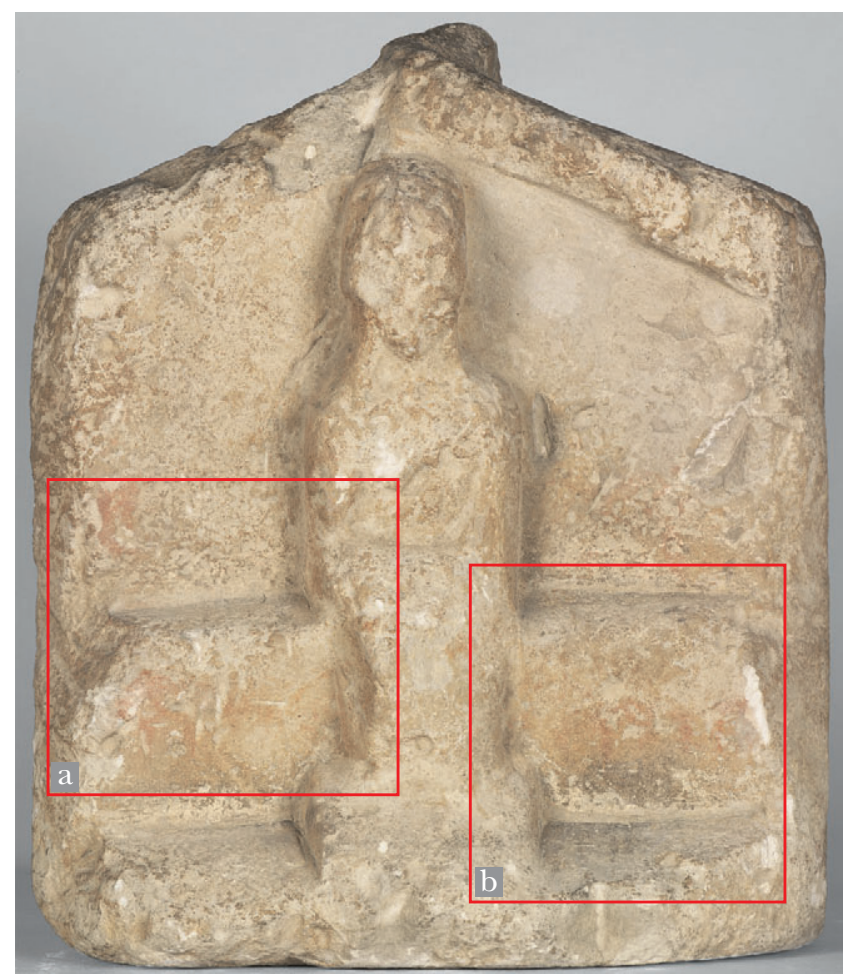

les éléments chimiques constitutifs du support et des restes de peinture. L'utilisation de la micro-diffraction des rayons $\mathrm{X}$ a contribué à préciser la nature des phases minérales présentes dans certaines couches ${ }^{11}$. La micro-spectrométrie Raman a ciblé plus précisément les grains de pigments.

\section{Résultats}

Les premières analyses par MFX, effectuées in situ sur les pièces sélectionnées, ont permis de restreindre la palette de pigments à la gamme des oxydes de fer ${ }^{12}$ pour les restes de couleur rouge et possiblement jaune ${ }^{13}$. Les grains de pigments
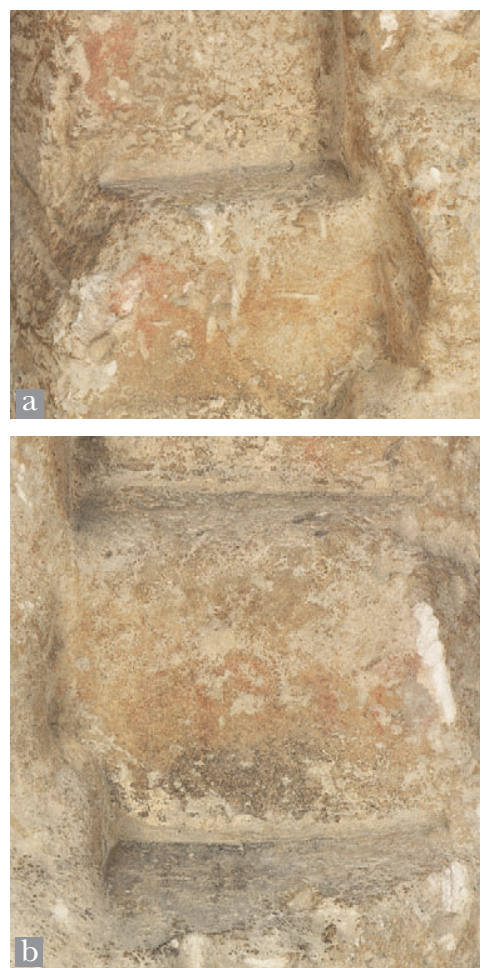

Fig. 7. Sur le naïskos inv. MHM. 83.7.588, restes de polychromie rouge dans le fond de la niche et sur la face antérieure de la banquette dans la partie supérieure (détail A) ; décor de franges peintes en rouge sur la face antérieure de la banquette dans la partie supérieure et restes de polychromie noire (et peut-être jaune ?) dans la partie inférieure (détail B). (c) O. Guillon.

noirs observés sur un prélèvement ont été identifiés par MEB/ EDS comme du noir de carbone en raison de l'absence de phosphore dans les grains analysés ${ }^{14}$.

À partir de l'analyse des coupes transversales de prélèvement, deux profils stratigraphiques différents ont été observés. Le premier présente, au-dessus du support calcaire, une première couche de faible épaisseur (environ $50 \mu \mathrm{m}$ ) majoritairement constituée d'argiles et contenant des grains de dolomite et de quartz. Les phases minérales muscovite et kaolinite identifiées par micro-diffraction des rayons X confirment la nature argileuse de ce qui pourrait être une couche de préparation brun clair à base de terre finement broyée. La couche colorée est constituée de pigments (ocre rouge et 

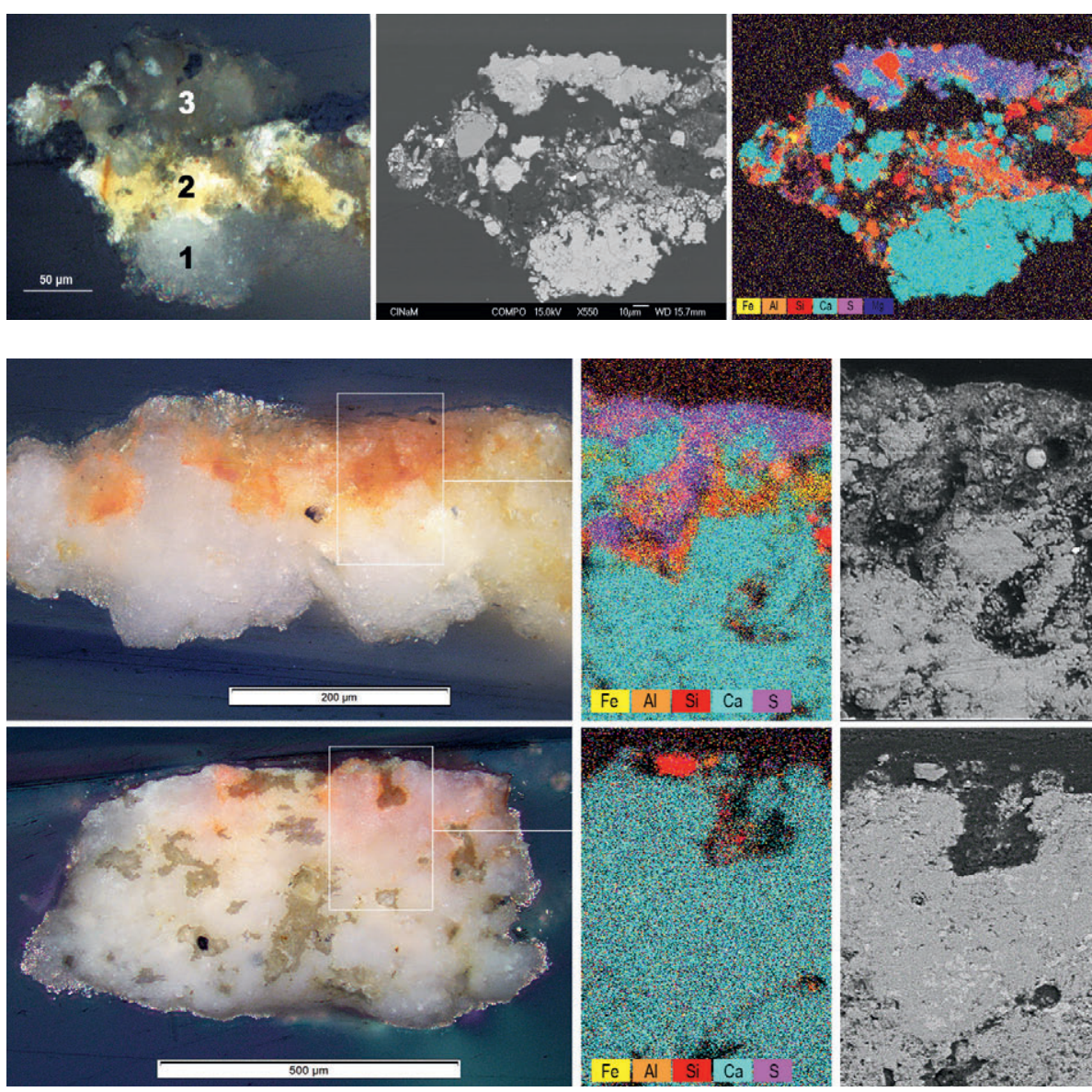

Fig. 8. Coupe stratigraphique d'un prélèvement de reste de polychromie noire. Mise en évidence du profil stratigraphique $\mathrm{n}^{\circ} 1$ sur le naïskos inv. MHM 83.7.588. On distingue 3 couches : 1. support de pierre calcaire en carbonate de calcium ; 2. couche préparatoire à base de terre argileuse contenant des grains de dolomite ; 3 . couche de couleur gris foncé contenant du gypse et des grains de noir de carbone. () N. Bouillon. noir de carbone identifiés) mélangés à du gypse ${ }^{15}$ et à des grains de calcite (fig. 8 et fig. 9, profil $n^{\circ} 1$ ). La répartition des particules colorées dans la matrice gypseuse laisse penser que le gypse a été utilisé comme liant hydraulique. Néanmoins, l'utilisation d'un liant organique ne peut être totalement exclue. Les analyses effectuées par CPG/SM ont révélé, dans un seul prélèvement de couleur, des traces de protéines, mais la très faible intensité du signal et l'absence d'acides aminés dans d'autres prélèvements du même type appellent à la prudence quant à cette hypothèse. Enfin, une fine couche plus pure en gypse et moins pigmentée s'est formée en surface. Cette couche ne résulte pas d'une contamination par le terrain d'enfouissement ou d'une néoformation liée au stockage des pièces à l'extérieur, car les naïskoi présentant le second profil stratigraphique n'en comportent pas. L'hypothèse d'une remobilisation en surface du liant gypseux de la couche sousjacente colorée apparaît comme la plus probable.

Le deuxième profil stratigraphique montre une application de la couche colorée directement sur le support en pierre calcaire. Les restes de peinture de ce type sont uniquement de couleur rouge et sont constitués d'un mélange de carbonate de calcium et d'oxydes de fer dénué de gypse (fig. 9, profil $\mathrm{n}^{\circ} 2$ ). La très faible quantité d'oxyde de fer dans la couche colorée surprend pour des restes de couleur qui apparaissent pourtant avec une teinte rouge plus vive. La distribution de grains d'oxyde de fer qui ne sont pas co-localisés avec l'aluminium et le silicium en cartographie X élémentaire indique l'utilisation d'hématite pure dont certains grains ont pu être isolés et identifiés au microscope électronique à transmission ${ }^{16}$. La répartition des pigments dispersés dans une matrice carbonatée et la difficulté de distinguer l'interface entre le support pierre et la couche colorée plaideraient plutôt en faveur de l'utilisation d'une technique à fresque ou d'une technique mixte où les pigments seraient mélangés à un lait de chaux ou bien étalés sur un enduit sec ré-humidifié au lait de chaux.

\section{Conclusion}

Les naïskoi sont sculptés dans un calcaire qui s'apparente à celui de Saint-Victor. Toutefois, l'absence de traces d'une exploitation antérieure à l'époque hellénistique ne permet pas d'affirmer que les carrières anciennes se situaient à l'emplacement exact de la future église, ainsi on préférera parler d'un calcaire « de type » Saint-Victor, d'autant que la formation affleure dans une large zone au sud et au nord du massif urgonien de Notre-Dame-de-la-Garde et à Saint-Giniez ${ }^{17}$.

L'analyse des couches peintes s'avère particulièrement difficile en raison du caractère extrêmement ténu des vestiges conservés. L'absence de données sur la composition du sol d'enfouissement, la quasi-méconnaissance des conditions de 
conservation et de traitement au cours de l'histoire matérielle de la collection limitent la compréhension des modifications physico-chimiques - d'origine naturelle ou anthropique - qui sont intervenues en surface des sculptures, et compliquent ainsi l'interprétation des données. Néanmoins, il semble bien que deux techniques différentes aient été utilisées. L'emploi d'une couche de préparation argileuse et de gypse dans la couche colorée, observés sur deux naïskoi, trouve un parallèle à Marseille à la même période : des fragments d'enduits peints sur maçonnerie de briques de terre crue, datés entre 510 et 450 avant J.-C., découverts dans les fouilles du collège du Vieux-Port en 2005, ont fait l'objet de prélèvements dont l'analyse scientifique a montré que les pigments ont été mélangés à du gypse et appliqués sur une couche de préparation à base de terre et chaux ${ }^{18}$.

L'observation de deux profils stratigraphiques différents - qui ne coexistent pas sur un même objet - est d'autant plus surprenante qu'ils ont été identifiés sur des naïskoi qui appartiennent pourtant à un même type stylistique. La quasi absence d'éléments de comparaison - à l'inverse des études sur la statuaire grecque archaïque en marbre, les analyses scientifiques sur celles en calcaire sont rares - ne permet pas de tirer de conclusions probantes sur ces choix techniques. Toutefois, à l'époque archaïque, l'emploi de plusieurs techniques d'application sur un même objet ne semble pas inconnue : l'analyse des pigments prélevés sur un chapiteau hathorique en calcaire découvert à Amathonte (Chypre), daté du début du V siècle avant J.-C., a montré que seuls le bleu et le noir étaient appliqués sur une couche préparatoire (peut-être du lait de chaux), tandis que celle-ci n'a pas été identifiée sous le rouge, une ocre à base d'oxydes de fer mêlée à de l'argile ${ }^{19}$.

Alors que certaines données restent difficiles d'interprétation, l'ensemble de ces résultats apportent toutefois une meilleure connaissance, d'une part, de l'application de la couleur sur la sculpture grecque en calcaire, et d'autre part, de l'artisanat de la sculpture en pierre à Marseille à l'époque archaïque, dont les naïskoi sont, à ce jour, les seuls témoignages.

\section{Remerciements}

Nous tenons à remercier le directeur du Musée d'Histoire de Marseille, L. Védrine, de nous avoir permis de mener à bien cette étude.

\section{Notes}

1. À ce propos, voir Hermary, 2000.

2. Cette collaboration a été mise en place dans le cadre de la thèse de doctorat de L. Rohaut, dans laquelle l'intégralité des résultats a été présentée (Rohaut, 2017). Les rapports d'analyse et le dossier d'imagerie scientifique sont disponibles au pôle scientifique du CICRP.

3. D’après J. Cantini, cité dans Clerc, 1927, voir Hermary, 2000, p. 120.

4. D'après R. Colomb en 1991 , voir Hermary, 2000, p. 120.

5. Gaudon, Nury, Tréziny, 2009.

6. Notice de la carte géologique 1/50000 BRGM n 1044, Aubagne-Marseille.

7. Clerc, 1927, p. 225. L'auteur fait mention de la présence de bleu et de rouge sur plusieurs exemplaires.

8. D’après les propos de J. Bouis, chargé de la restauration, recueillis lors d'un entretien en 1992 et retranscrits dans la maîtrise de M.-Fr. Pey-Rousset-Rouvière, soutenue la même année à l'Université de Provence. Voir Rohaut, 2017, p. 31.

9. Il s'agit d'un bactéricide et d'un anticryptogamique incolore à base de sels métalliques sans additifs chimiques.

10. Ces croûtes orange sont constituées de calcite, d'un oxyde de fer (probablement $\mathrm{Fe}_{2} \mathrm{O}_{3}$ ), montrant un gradient de concentration décroissant de la surface vers l'intérieur. L'hypothèse envisagée est le dépôt/diffusion des oxydes en solution à travers la porosité de la pierre. La présence de whewellite (oxalate de calcium) détectée en micro-diffraction des rayons $\mathrm{X}$ caractérise ces altérations et indique une possible action de micro-organismes.

11. Les analyses par microdiffraction des rayons X ont été effectuées par Vasile Heresanu du CINAM (Centre
Interdisciplinaire des Nanosciences de Marseille).

12. Absence de signal des éléments lourds à base de plomb (blanc de plomb, minium, litharge, massicot), de mercure (cinabre) ou d'arsenic (realgar, orpiment)

13. À l'inverse de l'hématite qui a pu être isolée et analysée au MET, la goethite n'a pu être identifiée de façon certaine.

14. Le phosphore entre dans la composition des noirs d'os et d'ivoire.

15. Co-localisation des éléments calcium et soufre dans les cartographies X des coupes stratigraphiques au MEB/EDS.

16. Les analyses par microscopie électronique à transmission ont été effectuées par Olivier Grauby du CINAM.

17. Cette position prudente s'est avérée judicieuse après la découverte récente, boulevard de la Corderie, d'une carrière de calcaire, de la $2^{\mathrm{e}}$ moitié ou de la fin du $\mathrm{VI}^{\mathrm{e}} \mathrm{s}$. av. J.-C. et du $1^{\text {er }}$ quart du $\mathrm{V}^{\mathrm{e}}$ s. av. J.-C. (fouilles préventives dirigées par Ph. Mellinand (INRAP) entre avril et juin 2017). Située en amont de l'abbaye, au pied du massif de la Garde, celle-ci relance la discussion sur la provenance de la pierre à l'époque archaïque et amène à reconsidérer l'hypothèse établie par les chercheurs qui consistent à localiser les carrières anciennes plus en avant de l'abbaye, au plus près du Lacydon.

18. Les analyses ont été effectuées en collaboration entre le CICRP, l'université de Provence Saint-Charles et le Service archéologique municipal-Atelier du Patrimoine de la ville de Marseille. Voir D'Ovidio, Bromblet, 2012.

19. L'étude a été faite par M. Schvoerer et son équipe au Centre de Recherches Interdisciplinaire d'Archéologie Analytique de Bordeaux : Schvoerer, Lamothe, Martinaud, 1985

\section{Bibliographie}

Clerc M., 1927, Massalia. Histoire de Marseille dans l'Antiquité des origines à la fin de l'empire romain d'Occident (476 ap. J.-C.), tome I. Des origines jusqu'au $I I I^{e}$ siècle av. J.-C., Marseille.

D’Ovidio A.-M., Bromblet Ph., 2012, « Les enduits peints du site archéologique du collège du Vieux-Port à Marseille (510450 av. J.-C.) : étude des pigments ", dans Fuchs M.-E., Monier F. (dir.), Les enduits peints en Gaule romaine: approches croisées. Actes du $23^{e}$ séminaire de l'Association Française pour la Peinture Murale Antique, Paris, ENS (13-14 novembre 2009), Revue archéologique de l'Est, Dijon, p. 247-250.

Gaudon P., Nury D., Tréziny H., 2009, «Les calcaires de Saint-Victor et leur utilisation dans Marseille antique et médiévale ", dans Fixot M., Pelletier J.-P., Saint-Victor de Marseille : étude archéologique et monumentale, Brepols, Turnhout, p. 9-16.

Hermary A., 2000, « Les naïskoi votifs de Marseille » dans Hermary A., Tréziny H. (dir.), Les cultes des cités phocéennes. Actes du colloque international Aix-enProvence/Marseille, 4-5 juin 1999, Edisud, Aix-en-Provence, p. 119-133.

Rohaut L., 2017, Les naïskoi votifs de Marseille. Étude des édicules avec femme assise dans les cités phocéennes, ioniennes et éoliennes à l'époque archaïque, thèse de doctorat soutenue le 9 juin 2017 à l'université d'Aix-Marseille.

Schvoerer M., Lamothe V., Martinaud M., 1985, « Appendice II. Étude physique des pigments rouge, bleus et noir du chapiteau ", BCH 109, p. 702-708. 